ORIGINAL ARTICLE

\title{
Emergency department intubation of trauma patients with undiagnosed cervical spine injury
}

\section{H Patterson}

Correspondence to: Dr H Patterson, Department of Emergency Medicine, Royal Perth Hospital, Box X2213 GPO, Perth WA 6001, Australia; harry.patterson@health. wa.gov.au

Accepted for publication 7 April 2003

\begin{abstract}
Objectives: Some trauma patients have an undiagnosed cervical spine injury but require immediate airway control. This paper reports an emergency department's (ED) experience with these patients. In particular, is there a worse neurological outcome?

Methods: A retrospective study over 6.5 years, based on prospectively collected data from the Royal Perth Hospital trauma registry. Patients with a cervical spine injury were identified and clinical data were abstracted. The primary outcome measure was evidence of exacerbation of cervical spine injury as a result of intubation by ED medical staff.

Results: 308 patients (1.9\%) of the 15747 trauma patients were intubated by ED medical staff. Thirty seven $(12 \%)$ were subsequently verified to have a cervical spine injury, of which 36 were managed with orotracheal intubation. Twenty five (69\%) survived to have a meaningful post-intubation neurological examination. Fourteen (56\%) of these 25 patients had an unstable cervical spine injury. Ninety per cent of all ED intubations were by ED medical staff. No worsening of neurological outcomes occurred.

Conclusions: Every ninth trauma patient that this ED intubates has a cervical spine injury. Intubation by ED medical staff did not worsen neurological outcome. In the controlled setting of an ED staffed by senior practitioners, patients with undiagnosed cervical spine injury can be safely intubated.
\end{abstract}

S ome trauma patients have an undiagnosed cervical spine injury, but require immediate airway control on arrival at the emergency department (ED). Intubation could potentially exacerbate the cervical spine injury, thus resulting in a worse neurological outcome.

The reported incidence of cervical spine injury in the setting of major trauma is $1.5 \%-4 \% .^{1-4}$ This rises to $7.8 \%$ in patients with a Glasgow coma scale (GCS) of less than $8 .^{5}$ It has been postulated that $3 \%-25 \%$ of patients with cervical spine injury may suffer injury extension as a consequence of delays in diagnosis, or inappropriate handling of their injury. ${ }^{6-8}$

Despite these concerns, recent studies have concluded that with meticulous technique by experienced practitioners, no worsening of neurological insult, or development of new deficit has occurred as a consequence of airway management. ${ }^{9-13}$ No study has investigated the issue of ED intubation by ED medical staff of undiagnosed cervical spine injury.

The aim of this study is to report our experience of trauma patients requiring intubation in the $\mathrm{ED}$, who have at that time, an undiagnosed cervical spine injury.

\section{METHODS}

This study was conducted at Royal Perth Hospital (RPH). $\mathrm{RPH}$ is an adult tertiary referral hospital and is the largest hospital in Western Australia. The hospital includes the state's spinal injuries unit. The ED has an annual census of some 54000 patients, with an admission rate of $44 \%$. The ED receives the majority of metropolitan and rural major trauma. $^{14}$ Approval for the study was granted by the hospital's trauma committee.

The ED is staffed by specialist emergency physicians, training registrars in emergency medicine, and junior medical staff. A team approach is used in the management of trauma, ${ }^{15}$ and the team leader is always a senior ED doctor. Typically, the airway is initially managed by the registrar in emergency medicine. All intubations were performed using a rapid sequence induction technique, using the orotracheal route.

All care is taken to minimise movement of the cervical spine, thus maintaining its normal anatomical alignment. There was however, no prospective recording of the immobilisation techniques used, and it is known that there is some variability between the approach used by individual emergency physicians. All trauma patients are managed on wooden spinal boards. Prehospital immobilisation collars are left in situ until we are ready to intubate. These prehospital devices are supported by the use of sandbags. Immobilisation is further increased by verbal reinforcement, if the patient is capable of understanding, and direct supervision. Just before induction, the cervical collar is loosened or removed. During laryngoscopy we endeavour to minimise extension of the cervical spine with a gentle atraumatic approach. We teach the application of in-line stabilisation as per ATLS guidelines, although staff may not always be available to perform this manoeuvre; hence it was not universally applied.

The hospital has a trauma registry that prospectively collects data for all trauma admissions $>24$ hours and all trauma deaths. A retrospective study was done for the period 1 July 1994 to 31 December 2000. The database was interrogated to identify patients who were intubated in the ED by ED medical staff, who were subsequently proved to have a cervical spine injury.

Excluded from this analysis were patients in whom it was not possible to perform a meaningful post-intubation neurological assessment. This includes patients who remained pharmacologically paralysed until brain stem death.

Once the subjects were identified, case notes were examined by trained abstracters using preformatted datasheets. The abstracters were blinded to the purpose of the study and interrater reliability was measured using Cohen's unweighted $\kappa$ score. ${ }^{16}$ 
Casenote review was necessary to:

- Confirm ED medical staff involvement

- Elicit pre-intubation neurology

- Establish periprocedural complications

- Determine final neurological outcome

The data collected included:

- Demographics

- Mechanism of injury

- Presence of cervical spine injury

- Stability of cervical spine injury. (For this study, the definition of stability was arrived at following the description in "Comprehensive classification for fractures" from the American Orthopedic Trauma Association. ${ }^{17}$

- Specialty and seniority of airway doctor

- Pre-intubation and post-intubation neurological evaluation

- Final outcome

- Trauma scoring

- Haemodynamic complications of intubation

The primary outcome measure was evidence of exacerbation of cervical spine injury as a result of endotracheal intubation by an ED doctor. The above data were collated and analysed.

\section{RESULTS}

There were 15747 patients registered on the trauma database during the study period. Of these 15747 patients, $432(2.7 \%$, $95 \%$ CI 2.5 to 3.0 ) had a cervical spine injury. Of these 432 patients $68(15.7 \%$, 95\%CI 12.3 to 19.2$)$ were intubated, either before arrival at the ED $(n=27)$, by ED medical staff $(n=37)$, or by anaesthetic staff $(n=4)$, within two hours of arrival (fig 1). The reasons for the anaesthetist intubations were: two patients became unstable while being escorted to the CT scan, one patient was assessed as a difficult intubation because of an expanding neck haematoma, and one was at the request of ED staff for an unspecified reason.

A total of $308(1.9 \%, 95 \%$ CI 1.7 to 2.2$)$ patients were intubated by ED medical staff. Of these 308 patients, 37 (12\%, 95\%CI 8.4 to 15.6 ) had an associated cervical spine injury. Of these 37 patients, 11 (30\%, 7 male) died without meaningful neurological examination post-intubation before death. All deaths occurred within 48 hours of arrival to the ED: six from uncontrolled bleeding, four from severe head injury, and one as a consequence of intractable ventricular fibrillation. One patient required a tracheostomy (because of maxillofacial trauma) as the first definitive airway procedure. This leaves a study population of 25 patients (with a preintubation neurological assessment) who were intubated by ED medical staff and had an undiagnosed cervical spine injury, in whom it was possible to perform a meaningful post-intubation neurological examination.

The mean age of these 25 patients was 37 years (range 1885 ), and 23 were male. The median injury severity ccore was 29 (range 8-75). Fourteen of the 25 patients (56\%) had unstable cervical spine injuries. Table 1 describes the mechanism of injury and distribution of stability.

Table 2 shows neurological presentation and outcome. There was no evidence of neurological deterioration as a consequence of ED intubation in any patient. No data were available for the number of intubation attempts, or the ease of intubation. Peri-intubation vital signs data were only available for 14 of the 25 patients. There were recorded transient changes in blood pressure in four patients: one

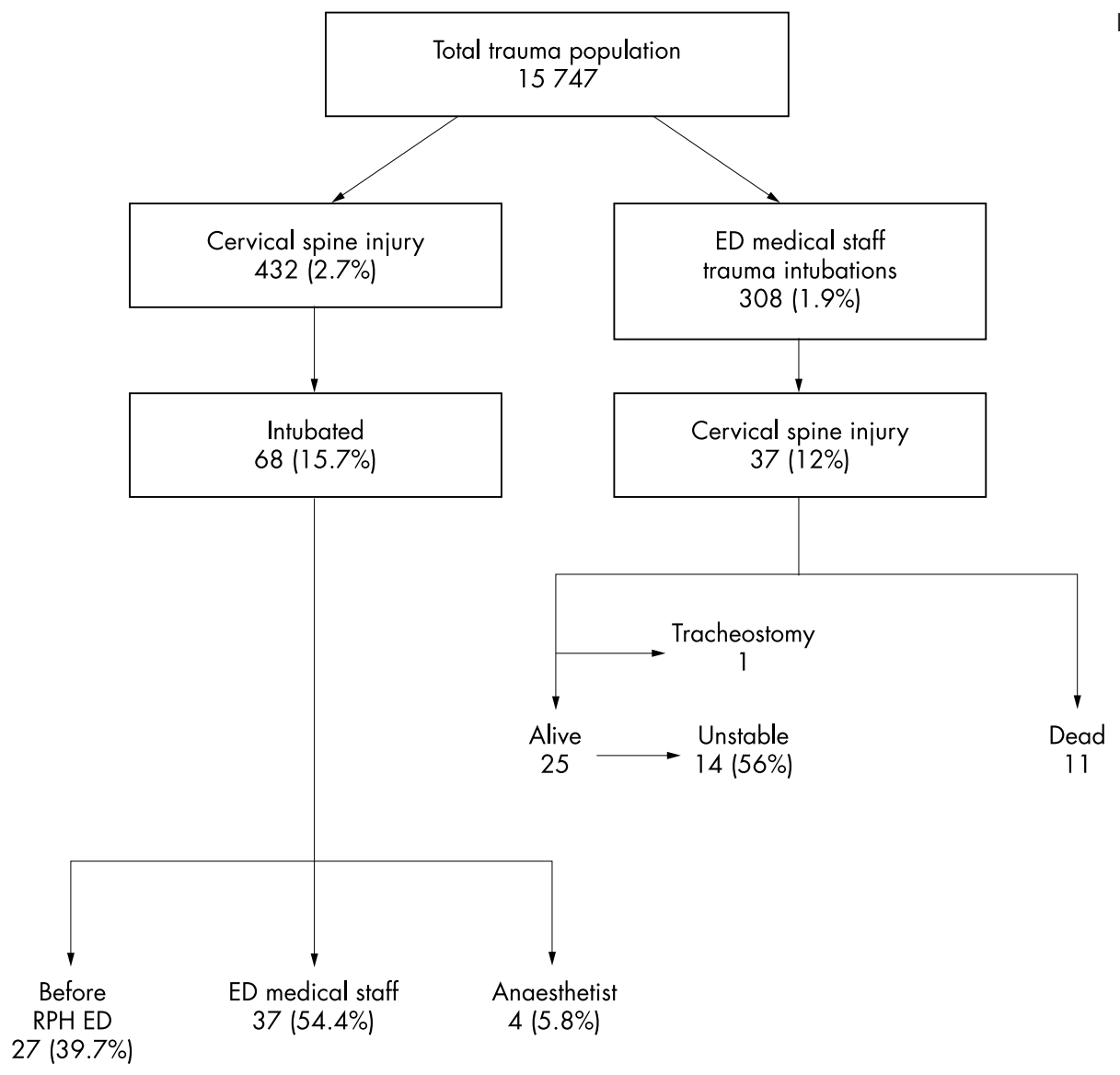

Figure 1 Outline of patient numbers. 
Table 1 Mechanism of injury for trauma patients with undiagnosed cervical spine injury, who were intubated by ED medical staff

\begin{tabular}{llll}
\hline Mechanism of injury & Stable cervical spine $(\mathbf{n})$ & Unstable cervical spine $(\mathbf{n})$ & Total \\
\hline Motor vehicle & 3 & 6 & 9 \\
Pedestrian & 4 & 3 & 7 \\
Motorcycle & 2 & 2 & 4 \\
Fall & 0 & 3 & 3 \\
Pedal cycle & 1 & 0 & 1 \\
Falling object & 1 & 0 & 1 \\
Total & $11(44 \%)$ & $14(56 \%)$ & $25(100 \%)$ \\
\hline
\end{tabular}

episode of hypotension, (SBP $87 \mathrm{~mm} \mathrm{Hg}$ ); and three patients with transient hypertension. These vital signs had returned to baseline within one to two minutes.

Twenty per cent of case notes were reviewed independently. Interrater reliability was calculated giving a $\kappa$ statistic of 0.91 .

\section{DISCUSSION}

We had no cases of a worsened neurological outcome after airway management by ED medical staff. In fact, ED medical staff intubated $90 \%$ of the trauma patients with a cervical spine injury who arrived at the ED requiring immediate airway control. This is significant when you consider that more than half the patients had an unstable cervical spine injury. Scannell et al reported a $71.6 \%$ rate of unstable injuries. ${ }^{11}$ However, stability was defined differently and emergency intubation was defined as within 24 hours, compared with our two hour definition. Patients who died were not included in our study, and this may have contributed to our lower rate of instability.

The most severe injury to the cervical spine probably occurs at the time of the injury. Therefore, the issue of stability may be less important than previously considered in relation to intubation of these patients. It is possible that only an uncontrolled approach to these patients will result in a worse neurological outcome.

There are only three published cases of a worsened neurological outcome after airway management. None involve the ED. Hastings and Kelly reported on a 65 year old man who required emergency intubation in the ICU by an anaesthetist, about 20 hours after the injury. ${ }^{18}$ The intubation was difficult and a cricothyroidotomy was performed. A short time later, there was no motor function in the lower extremities and weakness in the upper extremities. Imaging revealed extensive fractures at the level of C6-7. He was discharged to a chronic care facility.

Muckart et al reported the other two. ${ }^{19}$ A 45 year old man was involved in a motor vehicle accident. He suffered bilateral femoral fractures. After general anaesthesia for fixation of his injuries, he developed quadriplegia. Imaging revealed a fracture of $\mathrm{C} 2$, with subluxation of C2 on C3. A 22 year old man was admitted after multiple low velocity gunshot wounds to the neck, abdomen, and legs. After anaesthesia for a laparotomy, ventilation was by diaphragmatic movement alone and motor function was absent or reduced in all limbs. Imaging revealed a burst fracture of C6. A full recovery was made.

In these three cases, a cervical spine injury was neither detected or suspected, before intubation. This contrasts with

Table 2 Neurological presentation and outcome

\begin{tabular}{|c|c|c|c|c|c|c|}
\hline Age & Sex & Mechanism of injury & Entry neurology & ISS & $\mathrm{C} /$ spine stable & Outcome \\
\hline 28 & $M$ & MVA & L Brachial plexus & 45 & Yes & L plexopathy \\
\hline 85 & M & MVA/Train & GCS 6: limbs OK & 17 & No & Chronic head \\
\hline 46 & M & Ped/MVA & GCS 3 & 34 & Yes & Mild head \\
\hline 48 & M & Ped/MVA & GCS 3 & 29 & No & Chronic head \\
\hline 23 & M & MVA/Pole & Normal & 30 & Yes & Normal \\
\hline 23 & M & MVA/Pole & GCS 3 & 38 & No & Chronic head \\
\hline 39 & M & MBA/Tree & GCS 12: R arm flaccid & 14 & No & R plexopathy \\
\hline 62 & M & Fall $(4 \mathrm{~m})$ & Normal & 41 & No & Normal \\
\hline 18 & M & Ped/MVA & GCS 11: L arm weak & 14 & No & L plexopathy \\
\hline 48 & M & Fall $(7 \mathrm{~m})$ & GCS 3 & 29 & No & Mild head \\
\hline 61 & $\mathrm{~F}$ & Ped/MVA & GCS 5: limbs OK & 29 & Yes & Normal \\
\hline 26 & M & Ped/MVA & GCS 3 & 29 & Yes & Moderate head \\
\hline 26 & M & $M B A$ & GCS 12: limbs OK & 9 & Yes & Normal \\
\hline 40 & M & Ped/MVA & C5 quadriplegia & 43 & No & C5 quadriplegia \\
\hline 44 & M & MBA & Normal & 17 & Yes & Normal \\
\hline 23 & M & MVA & C3 quadriplegia & 75 & No & C3 quadriplegia \\
\hline 21 & M & Ped/Truck & GCS 3 & 22 & Yes & Normal \\
\hline 26 & M & MBA & GCS 13: limbs OK & 29 & No & Normal \\
\hline 37 & M & MVA & GCS 3 & 24 & No & Chronic head \\
\hline 40 & M & MVA (roll) & $\mathrm{R}$ brachial plexus & 41 & No & R plexopathy \\
\hline 24 & M & MVA & Normal & 24 & Yes & Normal \\
\hline 31 & M & Rock fall & Normal & 8 & Yes & Normal \\
\hline 34 & M & Fall $(4 \mathrm{~m})$ & C5 quadriplegia & 29 & No & C5 quadriplegia \\
\hline 18 & $\mathrm{~F}$ & MVA(?SAH) & GCS 8: limbs OK & 16 & No & Normal \\
\hline 72 & $M$ & Pedal cycle & GCS 12: R hemiplegia & 43 & Yes & Mild head \\
\hline
\end{tabular}

Stability refers to the ability of the cervical spine to maintain alignment under normal loading conditions. See methodology for definition. Plexopathy is neurological deficit in the upper limb attributable only to brachial plexus injury. The "head" outcome describes deficits as a result of head injury only: mild implies covert deficit, only demonstrable on formal testing: moderate implies overt deficit, but still independence for daily living: chronic head implies deficits that require ongoing support and supervision. Limbs OK statement indicates that the patient was moving all limbs, for neurological assessment. It does not address the issue of limb injury (see discussion on neurological examination). 
the current approach to trauma of always suspecting a cervical spine injury until proved otherwise.

No study to date has analysed neurological outcome after emergency intubation. However, some subgroup figures exist. Holby et al report on 9 of 133 patients who had no new neurological deficit after nasal intubation in the ED. ${ }^{13}$ Criswell and Parr report on 36 of 393 patients who had no new deficit after oral intubation in the ED. ${ }^{12}$ Both state that in controlled circumstances, patients with unstable cervical spine fractures can be safely intubated.

The clinical data published so far do not show any one particular technique for the intubation of patients with cervical spine injuries to be safer than any other. ${ }^{12}$ Indeed, it has been suggested that when performed with care, and in the hands of an experienced practitioner, the procedure of tracheal intubation in the patient with a cervical spine injury is a "low risk" intervention. ${ }^{12}$

In our trauma population, the prevalence of cervical spine injury was $2.7 \%$. This is similar to a number of existing reports, which range from $1.3 \%$ to $4 \%{ }^{20-24}$ The largest study of cervical spine injury reported a prevalence of $2.4 \% .^{24}$

More noteworthy is that $12 \%$ of all ED doctor intubations in trauma had a cervical spine injury. This means that every ninth trauma patient we intubate has a cervical spine injury. This is a higher figure than previous reports. Hills and Deane report that $7.3 \%$ of patients with a GCS $<8$ or who arrive intubated, have a cervical spine injury. ${ }^{5}$ Rhee et al reported a prevalence of $8.9 \%$ in anaesthetist intubated trauma patients in the ED. ${ }^{10}$

Inevitably, the neurological examination before life saving intubation is abbreviated. ${ }^{12}$ It only takes a few seconds to assess GCS, pupil responses, and limb movements. Within the time constraints of the resuscitation phase, this comparatively crude assessment provides a reasonably accurate assessment. ${ }^{12}$ We excluded patients in whom it was not possible to undertake a formal post-intubation assessment.

The limitations of this study are that it is retrospective and that the study population was small. Our approach to trauma may not be comparable to other centres, especially smaller institutions and systems in other countries. However, it would have external validity to institutions of similar size and workload in Australasia and other EDs that are adopting this model. It is also possible that the patients who died may have had an injury extension, however, it was not possible to assess for this because of the nature and extent of their injuries. The standard of care required for these patients (that is, immediate airway management) means that it is simply not possible to have a detailed pre-intubation neurological assessment. On these data, a sample size calculation on a $1 \%$ complication rate ${ }^{8}$ with $\alpha$ of 0.025 and $80 \%$ power, would require $8800 \mathrm{ED}$ doctor intubations to demonstrate safety.

In our 6.5 year experience, intubation in the ED of trauma patients with an undiagnosed cervical spine injury, by ED medical staff, did not result in a worsened neurological outcome. In the controlled setting of an ED staffed by senior practitioners, patients with undiagnosed cervical spine injury can be safely intubated.

\section{ACKNOWLEDGEMENTS}

The author is grateful for the support of the staff in the trauma registry, and to my colleagues in the ED. Special thanks to Dr Daniel Fatovich for his critical review of the manuscript.

\section{REFERENCES}

1 Hastings RH, Marks JD. Airway management for trauma patients with potential cervical spine injuries. Anesth Analg 1991;73:471-82.

2 Crosby ET, Lui A. The adult cervical spine: implications for airway management. Can J Anaesth 1990;37:77-93.

3 Roth BJ, Martin RR, Foley K, et al. Roentgenographic evaluation of the cervical spine: a selective approach. Arch Surg 1994;129:643-5.

4 Hoffman JR, Wolfson AB, Todd K, et al. Selective cervical spine radiology in blunt trauma: methodology of the national emergency $\mathrm{x}$-radiography utilisation study (NEXUS). Ann Emerg Med 1998;32:461-9.

5 Hills MW, Deane SA. Head injury and facial injury: is there an increased risk of cervical spine injury? J Trauma 1993;34:549-54.

6 Rogers WA. Fractures and dislocations of the cervical spine. J Bone Joint Surg 1957;39A:341

7 Bohlman HF. Acute fractures and dislocations of the cervical spine. J Bone Joint Surg 1979;61A: 1119-42.

8 Reid DC, Henderson R, Saboe L, et al. Etiology and clinical course of missed spine fractures. J Trauma 1987;27:980-6.

9 Meschino A, Devitt JH, Koch JP, et al. The safety of awake tracheal intubation in cervical spine injury. Can J Anaesth 1992;39:114-17.

10 Rhee KJ, Green W, Holcroft J, et al. Oral intubation in the multiply injured patient:the risk of exacerbating spinal cord damage. Ann Emerg Med 1990;19:51 1-14.

11 Scannell G, Waxman K, Tominaga G, et al. Orotracheal intubation in trauma patients with cervical fractures. Arch Surg 1993;128:903-6.

12 Criswell JC, Parr MJA. Airway management in patients with cervical spine injury. Anaesthesia 1994;49:900-3.

13 Holby J, Jordan R. Airway management in patients with unstable spine fractures. Ann Emerg Med 1989; 18:1237-9.

14 St John's Ambulance (W A). Annual report. Perth: St John's Ambulance (WA), 2000.

15 Myers CT, Brown AFT, Dunjey SJ, et al. Trauma teams: order from chaos. Emerg Med 1993;5:28-36.

16 Dawson B, Trapp RG. Basic and clinical biostatistics. 3rd edn. Boston: McGraw-Hill, 2001.

17 American Orthopaedic Trauma Association: Committee for Coding and Classification. Comprehensive classification for fractures. Rosemount, IL: AOTA, 1996.

18 Hastings RH, Kelley SD. Neurological deterioration associated with airway management in a cervical spine injured patient. Anesthesiology 1993;78:580-3.

19 Muckart DJJ, Bhagwanjee S, van der Merwe R. Spinal cord injury as a result of endotracheal intubation in patients with undiagnosed cervical spine fractures. Anesthesiology 1997;87:418-20.

20 Sinclair D, Swartz M, Gruss J, et al. A retrospective review of the relationship between facial fractures, head injuries and cervical spine injuries. J Emerg Med 1988;6:109-12.

21 Neifield GL, Keene JG, Hevesy G, et al. Cervical injury in head trauma. $J$ Emerg Med 1998;6:203-7.

22 Davidson JS, Birdsell DC. Cervical spine injury in patients with facial skeleton trauma. J Trauma 1989;29:1276-8.

23 Banit DM, Grace G, Fisher JR. Evaluation of the acute cervical spine: a management algorithm. J Trauma 2000;49:450-6.

24 Goldberg W, Meuller C, Panacek E et al, for the NEXUS group. Ann Emerg Med 2001;38:17-21. 